\title{
A PHASE RETRIEVAL METHOD FOR SIGNALS IN MODULATION-INVARIANT SPACES
}

Volker Pohl, Çă̆kan Yapar, Holger Boche

\author{
Lehrstuhl für Theoretische Informationstechnik \\ Technische Universität München \\ 80290 München, Germany \\ \{volker.pohl, cagkan.yapar, boche\}@tum.de
}

\author{
Fanny Yang
}

\author{
Department of EECS \\ University of California-Berkeley \\ Berkeley, CA 94720, USA \\ fanny-yang@berkeley.edu
}

\begin{abstract}
This paper considers the problem of signal recovery from magnitude measurements for signals in modulation invariant spaces. It proposes a measurement setup such that almost every signal in such a signal space can be reconstructed from its amplitude measurements up to a global constant phase and with a sampling rate of four times the rate of innovation of the signal space. The applicability of the proposed scheme under noise measurements is demonstrated by computer simulations.
\end{abstract}

Index Terms - Phase retrieval, sampling, modulation-invariant spaces, stationary sequences

\section{INTRODUCTION}

The problem of recovering a signal from magnitude measurements of its Fourier transform (also known as phase retrieval) arises in many different applications. In Fig. 1 the phase retrieval problem is sketched for a typical setup in optics (assume for the moment that the mask, shown in Fig. 1, is not present). The object of interest is illuminated by a light source. This produces a certain diffraction pattern $x(t)$, where $t$ stands for the spacial coordinate. This diffraction pattern contains the information about our object and the lens transform $x$ into the Fourier domain. There the intensity $|\widehat{x}(\omega)|^{2}$ of the Fourier transform $\widehat{x}(\omega)$ is measured and sampled on a certain regular grid with frequency spacing $\beta$. The phase retrieval problem is now to reconstruct the diffraction pattern $x(t)$ from the set of discrete intensity measurements $\left\{|\widehat{x}(n \beta)|^{2}\right\}_{n \in \mathbb{Z}}$.

Recently this phase retrieval problem has attracted some interest. For signals in finite dimensional spaces, [1] proposed an approach which applies a large number of intensity measurements to compensate for the loss of phase information. The paper derived necessary and sufficient conditions on the number on intensity measurements such that signal recovery becomes possible. Now it seems to be clear that in a $N$-dimensional space $4 N-4$ measurements are necessary and sufficient [2]. A related question is how to design the measurement vectors such that signal recovery is possible. In [3] a class of measurement vectors was proposed which allows for a simple analytic signal reconstruction. Nevertheless, $M=N^{2}$ measurements are needed in this case. Ideas from convex optimization where applied in $[4,5,6]$ for phaseless signal recovery using $\mathcal{O}(N \log N)$ random measurement vectors.

Only few results exist for infinite dimensional signal spaces. In [7] it was shown that real valued bandlimited function can be reconstructed from amplitude measurements taken uniformly at twice the

This work was partly supported by the German Research Foundation (DFG) under Grant BO 1734/22-1.

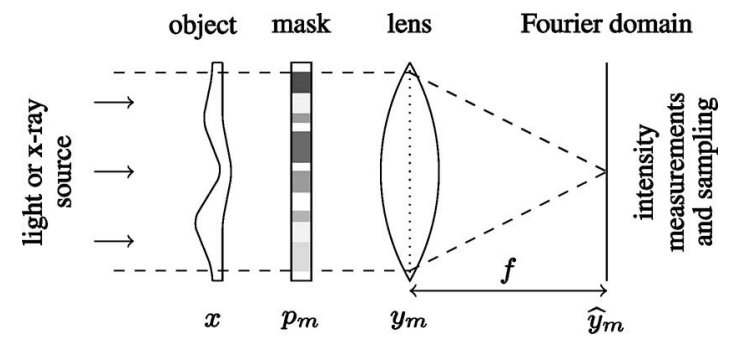

Fig. 1. A typical setup of a phase retrieval problem in optics.

Nyquist rate. However, this approach can not be extended to complex signals. In [8] it was shown that complex-valued, time (resp. spatially) limited signals can be reconstructed from amplitude measurements taken with a rate of at least 4 times the Nyquist rate and by applying particular structured modulations before signal sampling. Also a corresponding recovery procedure was proposed.

The assumption that the signal is perfectly time (or spatially) limited may be too restrictive in some situations and more flexible signal spaces are often desirable. Therefore, the present paper extends the approach from [8] to a larger signal class, namely to so called modulation-invariant spaces. It is shown that a sampling rate of four times the rate of innovation of the signal space is sufficient for perfect reconstruction. Numerical simulations will show that our approach is applicable also under disturbances of the measurements by additive noise.

\section{SIGNAL MODEL AND NOTATIONS}

Notations The Hilbert space of square integrable functions on the real axis $\mathbb{R}$, equipped with the usual inner product, is denoted by $L^{2}(\mathbb{R})$. For every $x \in L^{2}(\mathbb{R})$ its Fourier transform is given by

$$
\widehat{x}(\omega)=(\mathcal{F} x)(\omega)=\int_{\mathbb{R}} x(t) \mathrm{e}^{-\mathrm{i} \omega t} \mathrm{~d} t, \quad \omega \in \mathbb{R} .
$$

Let $\tau>0$ be arbitrary then the unitary translation and modulation operator on $L^{2}(\mathbb{R})$ is defined by

$$
\mathrm{T}_{\tau}: x(t) \mapsto x(t-\tau) \quad \text { and } \quad \mathrm{M}_{\tau}: x(t) \mapsto x(t) \mathrm{e}^{\mathrm{i} \tau t},
$$

respectively. In the following $\mathbb{T}$ stands for the interval $[0,2 \pi]$ on the real axis $\mathbb{R}$ and for any $1 \leq p \leq \infty$ we write $L^{p}(\mathbb{T})$ for the usual Lebesgue space of functions on $\mathbb{T}$. 
The Signal Space and its Characterization Let $g \in L^{2}(\mathbb{R})$ and $\tau>0$ be arbitrary. We consider signals in spaces of the form

$$
\mathcal{G}_{\tau}:=\overline{\operatorname{span}}\left\{g_{n}(t):=\left(\mathrm{M}_{\tau}^{n} g\right)(t)=g(t) \mathrm{e}^{\mathrm{i} n \tau t}: n \in \mathbb{Z}\right\}
$$

where the closure is taken in $L^{2}(\mathbb{R})$. Such spaces are usually called modulation-invariant subspace of $L^{2}(\mathbb{R})$ with generator $g$, and the sequence $g:=\left\{g_{n}\right\}_{n \in \mathbb{Z}}$ in $L^{2}(\mathbb{R})$ is the generator sequence of $\mathcal{G}_{\tau}$. It is not hard to see that $g$ forms a stationary sequence in $L^{2}(\mathbb{R})[9]$ and its correlation function has the spectral representation

$$
\left\langle g_{n}, g_{m}\right\rangle_{L^{2}(\mathbb{R})}=\frac{1}{2 \pi} \int_{\mathbb{T}} \mathrm{e}^{\mathrm{i}(n-m) \theta} \Phi_{g}(\theta) \mathrm{d} \theta
$$

with the spectral density $\Phi_{g}$, which is given by [9]

$$
\Phi_{g}(\theta)=\frac{2 \pi}{\tau} \sum_{k \in \mathbb{Z}}\left|g\left(\frac{\theta+k 2 \pi}{\tau}\right)\right|^{2}, \quad \theta \in \mathbb{T} .
$$

With the spectral density $\Phi_{g}$ of $g$ we associate the set

$$
\mathcal{M}_{g}:=\left\{\theta \in \mathbb{T}: \Phi_{g}(\theta)>0\right\} .
$$

It characterizes in some sense the redundancy of $\mathcal{G}_{\tau}$ [10]. We always assume that the generating sequence $g$ forms a frame for $\mathcal{G}_{\tau}$. Then every $x \in \mathcal{G}_{\tau}$ can be represented as

$$
x(t)=\sum_{n \in \mathbb{Z}} a_{n} g_{n}(t)=g(t) \sum_{n \in \mathbb{Z}} a_{n} \mathrm{e}^{\mathrm{i} n \tau t}
$$

with a coefficient sequence $a=\left\{a_{n}\right\}_{n \in \mathbb{Z}} \in \ell^{2}$. It can be shown that $g$ is a frame for $\mathcal{G}_{\tau}$ if there are positive constants $A, B$ such that

$$
A \leq \Phi_{g}(\theta) \leq B \quad \text { for almost all } \quad \theta \in \mathcal{M}_{g}
$$

and $\boldsymbol{g}$ is a Riesz basis for $\mathcal{G}_{\tau}$ if additionally the Lebesgue measure of $\mathcal{M}_{g}$ is equal to $2 \pi$ [9]. If $g$ is a frame but not a Riesz basis for $\mathcal{G}_{\tau}$ then $x \in \mathcal{G}_{\tau}$ does not uniquely determine the corresponding coefficient sequence $a$. However, the Fourier series $A(\theta)=$ $\sum_{n \in \mathbb{Z}} a_{n} \mathrm{e}^{\mathrm{i} n \theta}$ is uniquely determined on $\mathcal{M}_{\boldsymbol{g}}$, and the Fourier series associated with the so-called canonical coefficient sequence is characterized by the property that $A(\theta)=0$ for all $\theta \notin \mathcal{M}_{g}$.

Similarly, we may describe our signal space in the Fourier domain. Then the Fourier transform $\widehat{x}$ of every signal $x \in \mathcal{G}_{\tau}$ belongs to the shift-invariant space [11]

$$
\widehat{\mathcal{G}}_{\tau}:=\overline{\operatorname{span}}\left\{\widehat{g}_{n}(\omega):=\left(\mathrm{T}_{\tau}^{n} \widehat{g}\right)(\omega)=\widehat{g}(\omega-n \tau): n \in \mathbb{Z}\right\}
$$

where $\widehat{g}$ is the Fourier transform of the generator $g \in \mathcal{G}_{\tau}$. Therewith, the Fourier transform of the signal (3) has the form

$$
\widehat{x}(\omega)=\sum_{n \in \mathbb{Z}} a_{n} \widehat{g}_{n}(\omega)=\sum_{n \in \mathbb{Z}} a_{n} \widehat{g}(\omega-n \tau) .
$$

Following [12] we say that $\mathcal{G}_{\tau}$ has a rate of innovation of $\rho_{\mathcal{G}}=1 / \tau$.

For us it will not be sufficient that the generator of $\mathcal{G}_{\tau}$ belongs to $L^{2}(\mathbb{R})$. Instead we require that it satisfies the following condition.

Condition G: Let $g$ be a function on $\mathbb{R}$. We say that $g$ satisfies Condition $\mathrm{G}$ if there exist two constants $C>0$ and $\epsilon>0$ such that

$$
|g(t)| \leq C(1+|t|)^{-(1+\epsilon)} \quad \text { for all } t \in \mathbb{R} .
$$

Remark: If $g$ satisfies Condition $\mathrm{G}$ then $g \in L^{1}(\mathbb{R}) \cap L^{\infty}(\mathbb{R})$ which implies in particular that $g \in L^{2}(\mathbb{R})$.

Example 1: Let $T>0$ be arbitrary. Then the subspace $L^{2}([-T, T])$ of all $x \in L^{2}(\mathbb{R})$ with support in $[-T, T]$ is a modulation-invariant space (1) with generator

$$
g(t)=\frac{1}{\sqrt{2 T}} \chi_{[-T, T]}(t) \text { and } \tau \leq \frac{\pi}{T} .
$$

The spectral density, associated with this corresponding generator sequence, is then $\Phi_{g}(\theta)=\frac{\pi}{\tau T} \chi_{[-\tau T, \tau T]}(\theta)$, where $\chi_{\mathcal{T}}$ stands for the indicator function of the set $\mathcal{T}$.

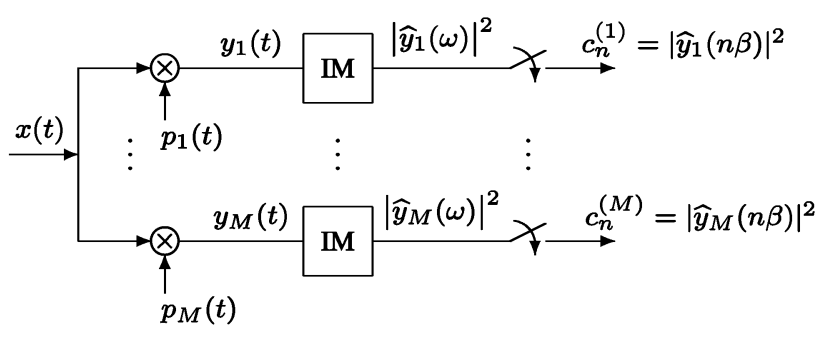

Fig. 2. Measurement setup with $M$ branches of modulators. A block with (IM) stand for Fourier transform and intensity measurement.

\section{STRUCTURED MODULATIONS}

In general, it is not clear whether it is possible to reconstruct a signal $x \in \mathcal{G}_{\tau}$ from the amplitudes of samples $|\widehat{x}(k \beta)|$ of its Fourier transform $\widehat{x}$ and if so, which sampling rate $1 / \beta$ is necessary. Results from finite dimensional phase retrieval $[1,2,4]$ even indicate that a simple (over) sampling of $|\widehat{x}(\omega)|$ is not sufficient for signal reconstruction. But rather more specific measurements are needed, i.e. that slightly more general measurement functionals have to be applied.

In optical applications such measurement functionals are obtained by inserting specific mask between the object and the lens, as sketched in Fig. 1. Each mask has a specific transmittance function $p_{m}(t)$ such that the signal samples obtained with mask $m$ become

$$
\left|\widehat{y}_{m}(n \beta)\right|=\left|\int_{\mathbb{R}} x(t) p_{m}(t) \mathrm{e}^{-\mathrm{i} n \beta t} \mathrm{~d} t\right|=\left|\left\langle x, s_{n}^{(m)}\right\rangle_{L^{2}(\mathbb{R})}\right|,
$$

where the functions $s_{n}^{(m)}(t):=p_{m}(t) \mathrm{e}^{-\mathrm{i} n \beta t}$ are determined by the mask and the sampling interval $\beta$. If the complex valued inner products $\left\langle x, s_{n}^{(m)}\right\rangle$ would be known then it would be fairly easy to determine a sequence $\left\{s_{n}^{(m)}\right\}$ such that every $x \in \mathcal{G}_{\tau}$ can be reconstructed [13]. However, if only the amplitudes of these inner products are known then it is not known which conditions the sequence $\left\{s_{n}^{(m)}\right\}$ has to satisfy such that signal recovery is always possible.

In [8] a specific choice of the masks $p_{m}$ was proposed such that every $x \in L^{2}([-T, T])$ can be recovered from its amplitude samples. It will be shown here that using the same masks and under some conditions on the generator $g$, every signal in any arbitrary modulation-invariant subspace of the form (1) can be reconstructed from its intensity measurements in the frequency domain.

Fig. 2 sketches schematically the setup of Fig. 1. The masks in Fig. 1 correspond now to a bank of $M$ modulators. It is assumed that these modulators have the following general form

$$
p_{m}(t):=\sum_{k=1}^{K} \overline{\alpha_{m, k}} \mathrm{e}^{\mathrm{i} \lambda_{k} t}
$$

where $\lambda_{k}$ and $\alpha_{m, k}$ are complex coefficients which are determined subsequently and where the bar denotes the complex conjugate. Assuming this form of the modulators, it is easily verified that for any $x \in L^{2}(\mathbb{R})$ the intensity measurements in Fig. 2 are given by

$$
\begin{aligned}
c_{n}^{(m)} & =\left|\widehat{y}^{(m)}(n \beta)\right|^{2}=\left|\sum_{k=1}^{K} \overline{\alpha_{m, k}} \widehat{x}\left(n \beta+\lambda_{k}\right)\right|^{2} \\
& =\left|\left\langle\widehat{\mathbf{x}}_{n}, \boldsymbol{\alpha}_{m}\right\rangle_{\mathbb{C}^{K}}\right|^{2}, \quad m=1, \ldots, M ; n \in \mathbb{Z} .
\end{aligned}
$$

with the length $K$ vectors

$$
\alpha_{m}:=\left(\begin{array}{c}
\alpha_{m, 1} \\
\vdots \\
\alpha_{m, K}
\end{array}\right) \quad \text { and } \quad \widehat{\mathbf{x}}_{n}:=\left(\begin{array}{c}
\widehat{x}\left(n \beta+\lambda_{1}\right) \\
\vdots \\
\widehat{x}\left(n \beta+\lambda_{K}\right)
\end{array}\right)
$$




\section{SIGNAL RECONSTRUCTION}

As in [8] the signal reconstruction is a three step procedure. In the first step, a finite number of signal samples of $\widehat{x}$ are recovered from the available amplitude measurements (7). In the second step, these finite blocks of $\widehat{x}$ are matched to eliminated unknown phase terms in each block. Finally the signal $x$ is obtained by interpolating $\widehat{x}$.

1. Finite Dimensional Phase Retrieval For every fixed $n \in \mathbb{Z}$ equations (7) constitute a $K$-dimensional phase retrieval problem for the unknown vector $\widehat{\mathbf{x}}_{n}$. It is known that one can find vectors $\boldsymbol{\alpha}_{m} \in \mathbb{C}^{K}$ such that $\widehat{\mathbf{x}}_{n}$ can be recovered up to a constant phase factor from the $M$ amplitude measurements $c_{n}^{(m)}$. In particular, if the measurement vectors $\alpha_{m}$ form a 2-uniform $M / K$-tight frame with $M=K^{2}$ vectors then every $\widehat{\mathbf{x}}_{n} \in \mathbb{C}^{K}$ can be reconstructed from the measurements (7) based on the following formula [1]

$$
\mathbf{Q}_{n}=\frac{1}{K} \sum_{m=1}^{M} c_{n}^{(m)}\left[(K+1) \boldsymbol{\alpha}_{m} \boldsymbol{\alpha}_{m}^{*}-\mathbf{I}_{K}\right]
$$

where $\mathbf{Q}_{n}=\widehat{\mathbf{x}}_{n} \widehat{\mathbf{x}}_{n}^{*}$ is a $K \times K$ matrix of rank 1 , and $\mathbf{I}_{K}$ stands for the $K \times K$ identity matrix. All values on the right hand side of (8) are known. Thus one can determine the matrix $\mathbf{Q}_{n}$ and factorize it as $\mathbf{Q}_{n}=\widehat{\mathbf{x}}_{n} \widehat{\mathbf{x}}_{n}^{*}$. This yields the vector $\widehat{\mathbf{x}}_{n}$ up to a constant phase factor, i.e. one obtains $\widehat{\mathbf{x}}_{n} \mathrm{e}^{\mathrm{i} \theta_{n}}$ where $\theta_{n}$ is an arbitrary phase which can not be determined due to the factorization of $\mathbf{Q}_{n}$.

2. Phase Propagation The different phase factors $\mathrm{e}^{\mathrm{i} \theta_{n}}$, obtained for each block $\widehat{\mathbf{x}}_{n}$, have to be matched because all blocks originate from the same overall signal $\widehat{x}$. To this end, we allow for an overlap between consecutive sets of interpolation points, i.e. we require that for every $n \in \mathbb{Z}$

$$
\left\{n \beta+\lambda_{k}\right\}_{k=1}^{K} \cap\left\{[n+1] \beta+\lambda_{k}\right\}_{k=1}^{K} \neq \emptyset .
$$

Clearly, such an overlap my be achieved by choosing

$$
\lambda_{K}=\lambda_{1}+\beta .
$$

Then $n \beta+\lambda_{K}=(n+1) \beta+\lambda_{1}$ for all $n \in \mathbb{Z}$, i.e. there is an overlap between consecutive vectors $\widehat{\mathbf{x}}_{n}$ in the sense that the last entry of $\widehat{\mathbf{x}}_{n}$ is equal to the first entry of $\widehat{\mathbf{x}}_{n+1}$.

Now we may start signal recovery at a certain index $n_{0} \in \mathbb{Z}$, determine $\widehat{\mathbf{x}}_{n_{0}}$, and set the phase $\theta_{n_{0}}$ arbitrary. In the next step we determine $\widehat{\mathbf{x}}_{n_{0}+1}$ and use the overlap between $\widehat{\mathbf{x}}_{n_{0}}$ and $\widehat{\mathbf{x}}_{n_{0}+1}$ to propagate the phase information from one vector to the next one. In this way, we are able to determine all vectors $\left\{\widehat{\mathbf{x}}_{n}\right\}_{n \in \mathbb{Z}}$ up to one unknown global phase factor [8]. At the end of this step, we know all vectors $\widehat{\mathbf{x}}_{n}$ which means that we know $\widehat{x}$ at the frequency points $n \beta+\lambda_{k}$ with $n \in \mathbb{Z}$ and $k=1, \ldots, K-1$.

3. Interpolation Now the frequency points $n \beta+\lambda_{k}$ have to be chosen such that every $x \in \mathcal{G}_{\tau}$ can be reconstructed from the known values of $\widehat{x}$ at these points. For simplicity of the presentation, we focus in this paper on the situation where the set of interpolation points is uniformly distributed on any horizontal axis of the complex plan. The following theorem considers the particular situation where

$$
\bigcup_{n \in \mathbb{Z}}\left\{n \beta+\lambda_{k}\right\}_{k=1}^{K}=\{m \tau\}_{m \in \mathbb{Z}}
$$

and it shows how $x \in \mathcal{G}_{\tau}$ can be reconstructed from such frequency measurements.
Theorem 1: Let $\mathcal{G}_{\tau}$ be a modulation invariant space of the form (1) with generator $g$ which satisfies Condition $G$. If there exists a constant $D>0$ such that

$$
G(\theta):=\left|\frac{2 \pi}{\tau} \sum_{k \in \mathbb{Z}} g\left(\frac{\theta+k 2 \pi}{\tau}\right)\right| \geq D \quad \text { for a.e. } \theta \in \mathcal{M}_{g} .
$$

Then every $x \in \mathcal{G}_{\tau}$ can recovered from its frequency samples $\{\widehat{x}(n \tau)\}_{n \in \mathbb{Z}}$ by

$$
x(t)=\sum_{n \in \mathbb{Z}} \widehat{x}(n \tau) \phi_{n}(t) \quad \text { with } \quad \phi_{n}(t)=\left(\mathrm{M}_{\tau}^{n} \phi\right)(t)
$$

and where the generator $\phi$ of the interpolation kernels is given by

$$
\phi(t)=\frac{\tau}{2 \pi} \frac{g(t)}{\sum_{k \in \mathbb{Z}} g\left(t+k \frac{2 \pi}{\tau}\right)} .
$$

Remark: In the definition of $\phi$ in (11) it should be understood that $\phi(t)=0$ for all $t \in \mathbb{R}$ where the denominator of the fraction on the right hand side is equal to zero.

Proof: Let $x \in \mathcal{G}_{\tau}$ be arbitrary. Since $\left\{g_{n}\right\}_{n \in \mathbb{Z}}$ is assumed to be a frame for $\mathcal{G}_{\tau}$ there exists a unique canonical coefficient sequence $a=\left\{a_{n}\right\}_{n \in \mathbb{Z}}$ such that $x$ can be written as in (3). By (4), we have

$$
\begin{aligned}
\widehat{x}(n \tau) & =\sum_{k=-\infty}^{\infty} a_{k} \widehat{g}([n-k] \tau) \\
& =\frac{1}{2 \pi} \int_{\mathbb{T}} A(\theta) G(\theta) \mathrm{e}^{-\mathrm{i} n \theta} \mathrm{d} \theta
\end{aligned}
$$

with $A(\theta)=\sum_{k \in \mathbb{Z}} a_{k} \mathrm{e}^{\mathrm{i} k \theta} \in L^{2}(\mathbb{T})$ and where we applied Poisson summation formula (cf. Lemma 3 in the Appendix) to obtain

$$
\sum_{k \in \mathbb{Z}} \widehat{g}(k \tau) \mathrm{e}^{\mathrm{i} k \theta}=\frac{2 \pi}{\tau} \sum_{k \in \mathbb{Z}} g\left(\frac{\theta+k 2 \pi}{\tau}\right)=: G(\theta) .
$$

Consequently (12) becomes

$$
X(\theta):=\sum_{n \in \mathbb{Z}} \widehat{x}(n \tau) \mathrm{e}^{\mathrm{i} n \theta}=A(\theta) G(\theta) .
$$

Since $G \in L^{\infty}(\mathbb{T})$ and $A \in L^{2}(\mathbb{T})$ it follows that $X \in L^{2}(\mathbb{T})$. Therefore the coefficient sequence $a$ can be determined from $\{\widehat{x}(n \tau)\}_{n \in \mathbb{Z}}$ by a linear filter

$$
a_{n}=\sum_{k \in \mathbb{Z}} \gamma_{k} \widehat{x}([n-k] \tau)
$$

with transfer function

$$
\Gamma(\theta)=\sum_{k \in \mathbb{Z}} \gamma_{k} \mathrm{e}^{\mathrm{i} k \theta}=\left\{\begin{array}{ccc}
1 / G(\theta) & \text { if } & \theta \in \mathcal{M}_{g} \\
0 & \text { if } & \theta \notin \mathcal{M}_{g}
\end{array}\right.
$$

where it was used that $a$ is the canonical coefficient sequence such that $A(\theta)=0$ for all $\theta \notin \mathcal{M}_{g}$. Finally, we plug (13) into (3) and a straightforward calculation gives (10).

To apply Theorem 1 we have to choose the coefficients $\lambda_{k}$ and the sampling period as follows:

$$
\begin{aligned}
\beta & =(K-1) \tau, \quad \lambda_{1}=\text { arbitrary }, \\
\lambda_{k} & =\lambda_{1}+(k-1) \frac{\beta}{K-1} \text { for } k=2,3, \ldots, K .
\end{aligned}
$$

Therewith the overlap condition (9) is satisfied and one gets

$$
\left\{\widehat{x}\left(n \beta+\lambda_{k}\right)\right\}_{n \in \mathbb{Z}}^{k=1, \ldots, K-1}=\left\{\widehat{x}\left(m \tau+\lambda_{1}\right)\right\}_{m \in \mathbb{Z}}=\{\widehat{u}(m \tau)\}_{m \in \mathbb{Z}}
$$

with $\widehat{u}(z):=\widehat{x}\left(z+\lambda_{1}\right)$. Then Theorem 1 can be applied to $\widehat{u}$. 
Overall Reconstruction Algorithm To summarize the whole procedure: To design the measurement system in Fig. 2 for a certain modulation invariant signal space $\mathcal{G}_{\tau}$ one has to

- Fix the degree $K \geq 2$ of the modulation functions (6).

- Determine $M=K^{2}$ vectors $\boldsymbol{\alpha}_{m}$ in $\mathbb{C}^{K}$ such that they form a 2-uniform $M / K$-tight frame (cf. [3, 14]). These vectors determine the coefficients $\alpha_{m, k}$ of the modulation functions (6).

- Choose the coefficients $\left\{\lambda_{k}\right\}_{k=1}^{K}$ of the modulation functions (6) and the sampling interval $\beta$ according to (14).

Then the reconstruction of any $x \in \mathcal{G}_{\tau}$ follows the following steps

1. At every sampling instant $n \in \mathbb{Z}$ one obtains the $M$ intensity measurements $\left\{c_{n}^{(m)}\right\}_{m=1}^{M}$ from the system in Fig. 2.

2. Determine the vectors $\widehat{\mathbf{x}}_{n}$ up to a constant phase factor $\mathrm{e}^{\mathrm{i} \theta_{n}}$ using (8) with a subsequent factorization of $\mathbf{Q}_{n}$.

3. Match the unknown phases over all $\widehat{\mathbf{x}}_{n}$ to obtain the set $\left\{\widehat{x}\left(m \tau+\lambda_{1}\right) \mathrm{e}^{\mathrm{i} \theta_{0}}\right\}_{m \in \mathbb{Z}}$ of signal samples.

4. Interpolate these points using Theorem 1 to reconstruct $x(t) \mathrm{e}^{\mathrm{i} \theta_{0}}$ up to a global constant phase factor $\mathrm{e}^{\mathrm{i} \theta_{0}}$.

The overall sampling rate (signal samples per frequency interval) is

$$
R=M \frac{1}{\beta}=\frac{K^{2}}{K-1} \frac{1}{\tau}=\frac{K^{2}}{K-1} \rho_{\mathcal{G}}
$$

which becomes minimal for $K=2$. Then it is $R_{\min }=4 \rho_{\mathcal{G}}$, i.e. four times the rate of innovation of the signal space $\mathcal{G}_{\tau}$.

Corollary 2: Let $\mathcal{G}_{\tau}$ be a modulation invariant space of the form (1) with generator $g$ which satisfies Condition $G$. Then every $x \in \mathcal{G}_{\tau}$ can be reconstructed from intensity measurements of $\widehat{x}$ taken at a rate of $R \geq R_{\min }=4 \rho_{\mathcal{G}}=4 / \tau$.

Example 2: A setting for the parameters $M, K, \beta$ and the coefficients $\lambda_{k}, \alpha_{m, k}$ in the setup of Fig.2 which achieves the minimal sampling rate $R_{\min }$, is given by $K=2, M=4, \beta=\tau$, and

$$
\alpha_{1}=\left(\begin{array}{l}
a \\
b
\end{array}\right), \quad \alpha_{2}=\left(\begin{array}{l}
b \\
a
\end{array}\right), \quad \alpha_{3}=\left(\begin{array}{c}
a \\
-b
\end{array}\right), \quad \alpha_{4}=\left(\begin{array}{c}
-b \\
a
\end{array}\right)
$$

with constants $a=\sqrt{\frac{1}{2}(1-1 / \sqrt{3})}$ and $b=\mathrm{e}^{\mathrm{i} 5 \pi / 4} \sqrt{\frac{1}{2}(1+1 / \sqrt{3})}$. Moreover $\lambda_{1}=0$ and $\lambda_{2}=\beta$ such that the vectors $\widehat{\mathbf{x}}_{n}$ become

$$
\widehat{\mathbf{x}}_{n}=[\widehat{x}(n \beta), \widehat{x}([n+1] \beta)]^{\mathrm{T}} \text {. }
$$

\section{NUMERICAL SIMULATIONS AND DISCUSSION}

To verify the applicability of our approach we performed numerical simulations. To this end, we fixed a modulation invariant signal space $\mathcal{G}_{\tau}$ with generator $g$ equal to a B-spline of order 2 [15] and with $\tau=3$. The Fourier transform of $g$ is known to be $\widehat{g}(\omega)=[\sin (\omega / 2) /(\omega / 2)]^{3}$. It easily follows that the spectral density $\Phi_{\mathbf{g}}(\theta)$ is strictly positive and bounded on $\mathbb{T}$ such that the sequence $\left\{g_{n}(t)=g(t) \mathrm{e}^{\mathrm{i} n \tau t}\right\}_{n \in \mathbb{Z}}$ is a Riesz basis for $\mathcal{G}_{\tau}$. Signals $x \in \mathcal{G}_{\tau}$ were produced by $x(t)=\sum_{n=-N}^{N} a_{n} g_{n}(t)$ from a sequence $\left\{a_{n}\right\}$ of independent, equally distributed, complex Gaussian random variables. In the frequency domain, the signal $\widehat{x}$ has the form (4) and because $\widehat{g}$ is mainly concentrated in the interval $[-2 \pi, 2 \pi]$, the bandwidth of $x$ is essentially equal to $\Omega_{x}=2[N \tau+2 \pi]$, i.e. it is basically proportional to the degree $N$. We applied the sampling setup as in Fig. 2 with parameters given in Example 2 and determined an estimate $\widetilde{x}(t)$ of $x(t)$, using the reconstruction procedure described above, from the noisy measurements

$$
c_{n}^{(m)}=\left|\widehat{y}_{m}(n \tau)\right|^{2}+\nu_{n}^{(m)}, \quad \begin{aligned}
& m=1, \ldots, M \\
& n=0, \pm 1, \ldots, \pm N_{\max },
\end{aligned}
$$

where $N_{\max }$ was chosen as the largest integer such that $\left|N_{\max } \tau\right|<$ $N \tau+2 \pi$, and where $\nu_{n}^{(m)} \sim \mathcal{N}\left(0, \sigma_{\nu}^{2}\right)$ are independent, normally distributed, complex random variables with variance $\sigma_{\nu}^{2}$. For every signal we determined the average power density of the signal

$$
\sigma_{s}^{2}=\frac{1}{4} \frac{1}{2 N_{\max }+1} \sum_{m=1}^{4} \sum_{n=-N_{\max }}^{N_{\max }}\left|\widehat{y}_{m}(n \tau)\right|^{2}
$$

and determined $\sigma_{\nu}^{2}$ such that a certain prescribed signal-to-noise ratio (SNR) $\varrho=\sigma_{s}^{2} / \sigma_{\nu}^{2}$ at the detector is achieved. After signal reconstruction, the relative means square error (MSE) is determined as $M S E=\|x-\widetilde{x}\|_{2}^{2} /\|x\|_{2}^{2}$. Therein, we substituted the correct phase from $x(t)$ for the unknown constant phase factor associated with $\widetilde{x}$. Simulation results where averaged over $10^{5}$ random signals $x \in \mathcal{G}_{\tau}$.

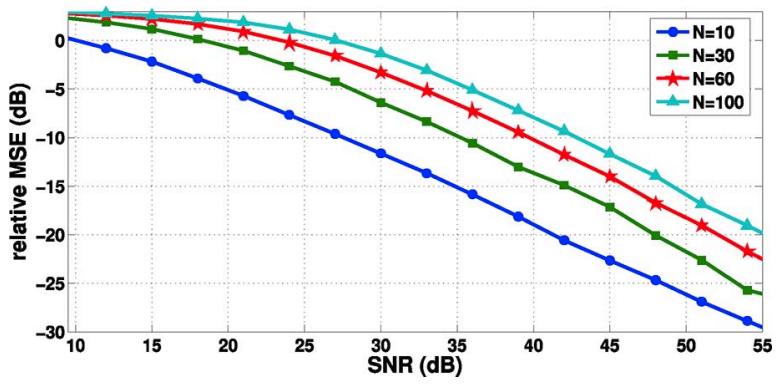

Fig. 3. Simulation results for signals $x$ with different degrees $N$, i.e. for signals with different bandwidths $\Omega_{x}=2[N \tau+2 \pi]$.

Fig. 3 shows the relative MSE versus SNR for different signal degrees $N$. It verifies that the proposed method basically works and recovers perfectly any signal from $\mathcal{G}_{\tau}$ as the SNR goes to infinity. Nevertheless, the simulation results also show that the relative MSE grows proportional with $N$, i.e. proportional with the number of measurements taken in the frequency domain.

In future research, it is an interesting question whether the MSE can be improved by the application of different reconstruction schemes or whether the stability behavior is already determined by the measurement setup.

\section{APPENDIX A - POISSON SUMMATION FORMULA}

Lemma 3: Let $g$ be a function on $\mathbb{R}$ which satisfies Condition $G$ and let $\tau>0$ be arbitrary. Then we have

$$
G(\theta):=\sum_{k \in \mathbb{Z}} \widehat{g}(k \tau) \mathrm{e}^{\mathrm{i} k \theta}=\frac{2 \pi}{\tau} \sum_{k \in \mathbb{Z}} g\left(\frac{\theta+k 2 \pi}{\tau}\right)
$$

for almost all $\theta \in \mathbb{T}$ and $G \in L^{\infty}(\mathbb{T})$.

Proof: Condition $\mathrm{G}$ implies that $g \in L^{1}(\mathbb{R})$ and that the function on the right hand side of (16), i.e.

$$
F(\theta):=\frac{2 \pi}{\tau} \sum_{k \in \mathbb{Z}} g\left(\frac{\theta+k 2 \pi}{\tau}\right), \quad \theta \in \mathbb{T}
$$

belongs to $L^{\infty}(\mathbb{T}) \subset L^{2}(\mathbb{T})$. Consequently, we can write $F$ as a Fourier series $F(\theta)=\sum_{n \in \mathbb{Z}} f_{n} \mathrm{e}^{\mathrm{i} n \theta}$ with Fourier coefficients

$$
\begin{aligned}
& f_{n}=\frac{1}{2 \pi} \int_{0}^{2 \pi} F(\theta) \mathrm{e}^{-\mathrm{i} n \theta} \mathrm{d} \theta=\sum_{k \in \mathbb{Z}} \frac{1}{\tau} \int_{0}^{2 \pi} g\left(\frac{\theta+k 2 \pi}{\tau}\right) \mathrm{e}^{-\mathrm{i} n \theta} \mathrm{d} \theta \\
& =\frac{1}{\tau} \int_{-\infty}^{\infty} g\left(\frac{\xi}{\tau}\right) \mathrm{e}^{-\mathrm{i} n \theta} \mathrm{d} \xi=\int_{-\infty}^{\infty} g(\omega) \mathrm{e}^{-\mathrm{i} n \tau \theta} \mathrm{d} \omega=\widehat{g}(n \tau)
\end{aligned}
$$

using that by Fubini's theorem the sum and integral can be exchanged since $g \in L^{1}(\mathbb{R})$. Thus, we obtained (16). 


\section{REFERENCES}

[1] R. Balan, P. G. Casazza, and D. Edidin, "On signal reconstruction without phase," Appl. Comput. Harmon. Anal., vol. 20, no. 3, pp. 345-356, May 2006.

[2] A. S. Bandeira, J. Cahill, D. G. Mixon, and A. A. Nelson, "Fundamental Limits of Phase Retrieval," in Proc. 10th Intern. Conf. on Sampling Theory and Applications (SampTA), Bremen, Germany, Jul. 2013.

[3] R. Balan, B. G. Bodmann, P. G. Casazza, and D. Edidin, "Painless reconstruction from magnitudes of frame coefficients," J. Fourier Anal. Appl., vol. 15, no. 4, pp. 488-501, Aug. 2009.

[4] E. J. Candès, Y. C. Eldar, T. Strohmer, and V. Voroninski, "Phase retrieval via matrix completion," SIAM J. Imaging Sci., vol. 6, no. 1, pp. 199-225, 2013.

[5] E. J. Candès, T. Strohmer, and V. Voroninski, "Phase Lift:Exact and Stable Signal Recovery from Magnitude Measurements via Convex Programming," Comm. Pure Appl. Math., vol. 66, no. 8, pp. 1241-1274, Aug. 2013.

[6] B. Alexeev, A. S. Bandeira, M. Fickus, and D. G. Mixon, "Phase Retrieval with Polarization," SIAM J. Imaging Sci., vol. 7, no. 1, pp. 35-66, 2014.

[7] G. Thakur, "Reconstruction of bandlimited functions from unsigned samples," J. Fourier Anal. Appl., vol. 17, no. 4, pp. 720 732, Aug. 2011

[8] F. Yang, V. Pohl, and H. Boche, "Phase retrieval via structured modulations in Paley-Wiener spaces," in Proc. 10th In tern. Conf. on Sampling Theory and Applications (SampTA), Bremen, Germany, Jul. 2013.

[9] T. Michaeli, V. Pohl, and Y. C. Eldar, "U-Invariant Sampling: Extrapolation and Causal Interpolation from Generalized Samples," IEEE Trans. Signal Process., vol. 59, no. 5, pp. 20852100, May 2011.

[10] V. Pohl, E. Tampubolon, and H. Boche, "Sampling and Reconstruction in Sparse Atomic Spaces," in Proc. 38th Intern. Conf. on Acoustics, Speech, and Signal Processing (ICASSP), Vancouver, Canada, May 2013.

[11] A. Aldroubi and K. Gröchenig, "Non-uniform sampling and reconstruction in shift-invariant spaces," SIAM Review, vol. 43, no. 4, pp. 585-620, 2001.

[12] M. Vetterli, P. Marziliano, and T. Blu, "Sampling Signals With Finite Rate of Innovation," IEEE Trans. Signal Process., vol. 50, no. 6, pp. 1417-1428, Jun. 2002.

[13] V. Pohl and H. Boche, "U-Invariant Sampling and Reconstruction in Atomic Spaces with Multiple Generators," IEEE Trans. Signal Process., vol. 60, no. 7, pp. 3506-3519, Jul. 2012.

[14] G. Zauner, "Quantum Designs: Foundations of a Noncommutative Design Theory," Int. J. Quantum Inform., vol. 9, no. 1, pp. 445-507, Feb. 2011.

[15] M. Unser, A. Aldroubi, and M. Eden, "B-spline signal processing. Part I \& II,” IEEE Trans. Signal Process., vol. 41, no. 2, pp. 821-848, Feb. 1993. 